\title{
Trois éléments de parure de l'Antiquité tardive de la villa des Bossenno à Carnac (Morbihan)
}

Three Late Roman artefacts from the Bossenno Villa in Carnac (Morbihan)

Tres piezas de ornamento de la Antigüedad tardía de la villa de Bossenno en

Carnac (Morbihan)

Drei Fundobjekte der römischen Militärausstattung spätantiker Zeit aus der

Villa von Bossenno bei Carnac (Morbihan, Bretagne)

\section{Barry Ager et Patrick Galliou}

\section{OpenEdition}

Journals

Édition électronique

URL : https://journals.openedition.org/rao/7937

DOI : $10.4000 /$ rao. 7937

ISSN : 1775-3732

Éditeur

Presses universitaires de Rennes

Édition imprimée

Date de publication : 21 décembre 2021

Pagination : 219-231

ISBN : 978-2-7535-8668-0

ISSN : 0767-709X

Référence électronique

Barry Ager et Patrick Galliou, «Trois éléments de parure de l'Antiquité tardive de la villa des Bossenno à Carnac (Morbihan)», Revue archéologique de l'Ouest [En ligne], 37 | 2021, mis en ligne le 21 décembre 2021, consulté le 13 octobre 2022. URL : http://journals.openedition.org/rao/7937 ; DOI : https:// doi.org/10.4000/rao.7937 


\title{
Trois éléments de parure de l'Antiquité tardive de la villa des Bossenno à Carnac (Morbihan)
}

\author{
Three Late Roman artefacts from the Bossenno Villa in Carnac (Morbihan)
}

\author{
Barry Ager \\ Curator, British Museum, department of Prehistory and Europe (ER) \\ Patrick Galliou \\ Professeur émérite, université de Bretagne occidentale (Centre de recherche bretonne et celtique)
}

\begin{abstract}
Résumé : L'étude de trois objets de cuivre allié (éléments de cingulum, fibule penannulaire) découverts au XIX ${ }^{e}$ s. dans la villa des Bossenno à Carnac (Morbihan) les place sans conteste dans l'Antiquité tardive (fin du Iv ${ }^{\mathrm{e}}$ s.-début du v ve.). On s'interrogera sur la place de ces artéfacts, ordinairement classés parmi les militaria, dans un habitat purement civil.
\end{abstract}

\begin{abstract}
Three copper alloy artefacts (cingulum elements, penannular brooch) discovered in the $19^{\text {th }}$ century in the Bossenno villa (Carnac, Morbihan) clearly belong to the Late Roman period (end of the $4^{\text {th }}$ century-early $5^{\text {th }}$ century). As such objects are commonly considered as militaria, their being found in an exclusively civilian settlement must be examined.
\end{abstract}

Mots clés : alliage cuivre, villa romaine, Antiquité tardive, Militaria.

Keywords: copper alloy, roman Villa, Late Roman period, Militaria.

Le nom de l'archéologue écossais James Miln (18191881) $)^{1}$ est lié, en Bretagne, à la fouille de sites de nature diverse, tant pré- et protohistoriques (Luco, 1881; Luco, 1883, par exemple) que d'époque romaine, de la commune de Carnac (Morbihan) [fig. 1]. Le plus connu de ces derniers est indiscutablement la villa des Bossenno, dans la partie méridionale du territoire communal, ensemble bâti que Miln explora entre 1874 et 1876 et qui

1. Fils de James Maud Miln, de Woodhill (Angus, Écosse), il entra dans la Royal Navy et participa, en Chine, à la première guerre de l'opium (1839-1842), puis commerça en Chine, en Inde et en Nouvelle-Zélande. Au décès de son père, il revint en Écosse, héritant de terres à Munrie et Woodhill, qui lui assurèrent un revenu confortable. Il put ainsi se livrer à ses passions pour les armes, l'astronomie, la photographie et l'archéologie, devenant Fellow de la Scottish Society of Antiquaries. Désireux de comparer les mégalithes écossais à ceux de Bretagne, il se rendit en LoireAtlantique et dans le Morbihan en 1873, explorant les monuments de la région en compagnie de son ami, le contre-amiral F. S. Tremlett, archéologue amateur et auteur d'un petit ouvrage sur le site mégalithique d'Er-Lannic, paru à Londres en 1884, puis s'installa à Carnac, fouillant divers sites de la commune. Membre de la Société française d'archéologie et de la Royal Northern Society of Antiquaries de Copenhague, il fut élu vice-président de la Société polymathique du Morbihan en 1881. Lors d'un voyage à Édimbourg, en 1881, il décéda de la typhoïde. fit l'objet, dès 1877, d'une double publication, en anglais et en français (Miln, 1877). Implantée non loin des eaux de la baie de Quiberon sur un site déjà occupé à la fin de La Tène (nombreux fragments de céramique commune et d'amphores vinaires Dressel IA et B), la villa comprenait au moins huit édifices (habitation, thermes, petit fanum privé, bâtiments à usage agricole), l'ensemble étant sans doute entouré d'un mur d'enceinte (fig. 2). Le mobilier recueilli lors de ces fouilles, couvrant une période allant du début du I ${ }^{\text {er }}$ s. apr. J.-C. à la fin du Iv s., fut légué à la ville de Carnac à la mort de Miln, puis déposé au musée que son frère Robert fonda dans la même ville ${ }^{2}$.

Dans ce mobilier, qui mériterait une étude détaillée à la lumière du renouvellement généralisé des identifications (matériaux, types, provenances, etc.) depuis le $\mathrm{XIX}^{\mathrm{e}}$ s., nous avons isolé trois pièces en alliage de cuivre qui, en raison

2. L'ancien musée (rue du Tumulus) fut bâti à partir de 1882. Robert Miln lui légua les collections de son frère, cataloguées dès 1894 (Catalogue du Musée ). Miln à Carnac (Morbihan), Vannes, Le Beau, 1894). 
Figure 1 : Carnac : plan de situation.

Figure 1: Carnac: location of the site.

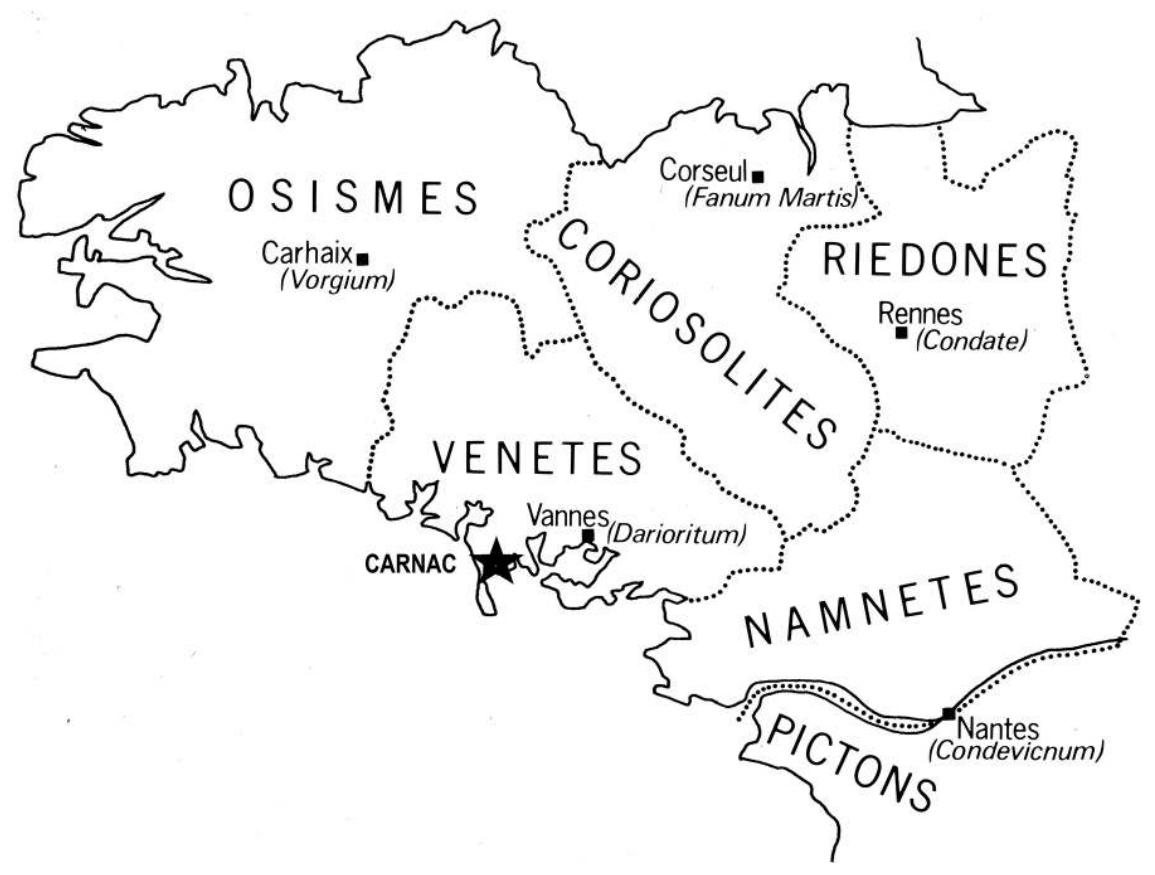

de leur singularité, méritent un examen approfondi. Ces objets, comme on le constatera, apportent d'intéressants renseignements sur l'occupation tardive de certains établissements ruraux ainsi que sur la question controversée du sens à donner à la présence d'objets de type "militaire " dans l'Armorique de la fin l'époque romaine.

\section{1. ÉLÉMENT DE PLAQUE-BOUCLE AJOURÉE (?) DU STYLE AMIENS-ANDOVER (FIG. 3)}

La première de ces pièces fut mise au jour dans la salle $\mathrm{n}^{\circ} 4$ du bâtiment $\mathrm{E}$ (Miln, 1877, p. 166 et fig. III, n $\left.{ }^{\circ}\right)^{3}$. Cet élément ajouré, présentant deux anneaux latéraux, pourrait être une contre-plaque de cingulum $^{4}$, un passecourroie $^{5}$ ou la moitié conservée d'une attache de ceinture, mais on ne connaît à ce jour aucun autre objet qui lui soit parfaitement identique. Bien qu'il soit endommagé et incomplet, on y reconnaît trois traits structuraux distinctifs qui le relient à un petit groupe - mais qui s'étoffe au gré de nouvelles découvertes - d'éléments de ceintures de l'Antiquité tardive, qui se rencontrent à la fois dans le

3. Miln la décrit comme suit : « Un ornement de bronze de destination inconnue, ayant peut-être fait partie d'une agrafe. Cette pièce intéressante, d'un travail remarquable, se compose de trois tiges plates se réunissant au sommet d'un triangle équilatéral, dont chaque angle est terminé par un cercle orné de deux têtes de dragon. La base est formée d'une large barre découpée en petits cercles ouverts. Cette pièce est ornée de traits et de points gravés en creux. " 4. Ceinturon de cuir souple sur lequel étaient fixés divers éléments utilitaires (boucle de fermeture) et décoratifs en alliage de cuivre.

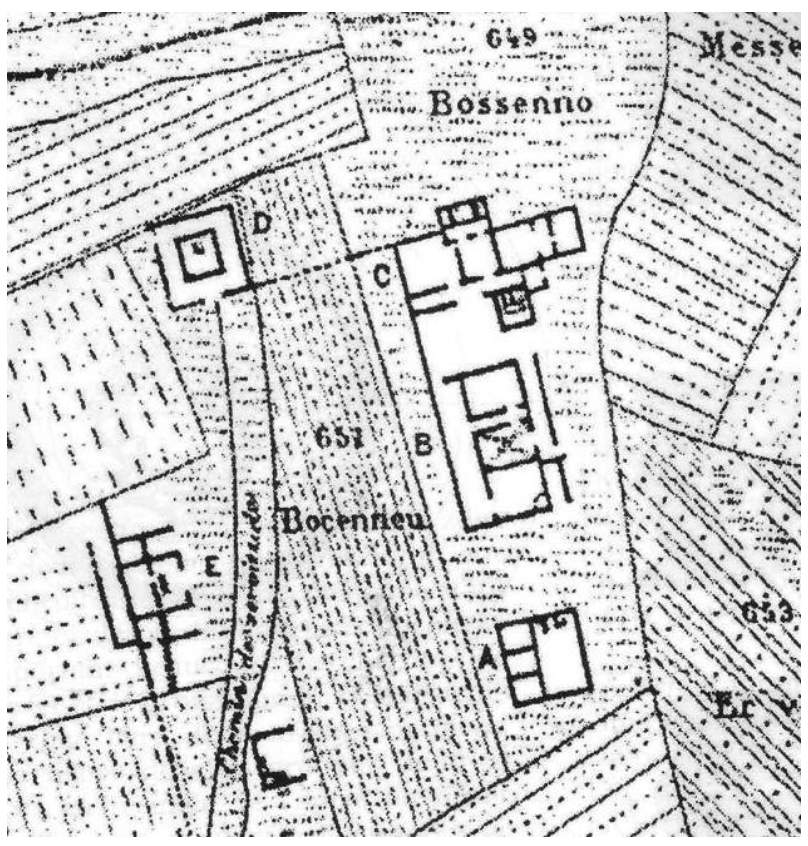

Figure 2 : Plan de la villa des Bossenno à Carnac (d'après J. Miln, 1877).

Figure 2: Plan of the Villa des Bossenno at Carnac (after J. Miln, 1877).

sud de l'Angleterre et dans le nord de la France. Ces traits sont les suivants :

- la forme triangulaire recoupée (soit la moitié d'un losange);

- l'anille centrale, en forme de croix à bras égaux;

- la présence en saillie, de part et d'autre de la pièce, de têtes animales à la gueule ouverte, présentées en paires affrontées. 


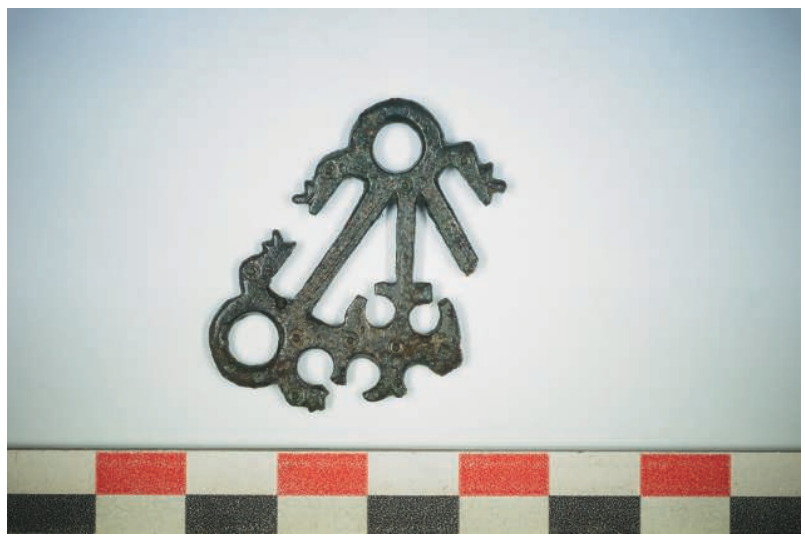

Figure 3 : Carnac, Les Bossenno : élément de cingulum du groupe AmiensAndover (cliché : M. Pérez-Bleuzen, Musée de Carnac).

Figure 3: Carnac, Les Bossenno: element of a cingulum of the AmiensAndover group (Photo: M. Pérez-Bleuzen, Musée de Carnac).

Ce groupe fut bien mis en évidence en 1985 par Alison Cook dans son analyse stylistique de la plaque-boucle de ceinture à décor ajouré - la boucle et la plaque sont d'un seul tenant -, découverte à hauteur de la taille dans une tombe de femme (sépulture $n^{\circ} 50$ ), datée de la fin du $v^{\mathrm{e}} \mathrm{s}$. ou du début du siècle suivant, du cimetière anglo-saxon de Portway à Andover (Hampshire, Grande-Bretagne) [fig. 4]. Pour Alison Cook, cette plaque est apparentée à une autre plaque-boucle, anciennement découverte à Amiens, dans des circonstances et un environnement archéologique inconnus, et aujourd'hui conservée à l'Ashmolean Museum d'Oxford (fig. 5), ainsi qu'à une courte série d'autres objets relevant du même style (Cook et Dacre, 1985, p. 96, pl. 17, fig. 63; Evison, 1981, p. 134, pl. 11a; MacGregor, 1997, p. 155, n 77.5; Suzuki 2000 , p. $128-129$, n $^{\circ} 5$, fig. 5 , pl. 5 , et p. 132-133, n ${ }^{\circ}$, fig. 9) ${ }^{6}$. Cet ensemble est connu sous le nom de " groupe d'Amiens-Andover ", d'après le lieu de découverte des deux principales trouvailles.

Mais, à y regarder de plus près, il convient, en fait, de distinguer deux sous-ensembles dans ce groupe : a) "type Amiens ", avec une anille cruciforme centrale; b) " type Andover », avec, au centre, un élément quadrilobé cruciforme, composé de quatre anneaux ajourés. L'objet des Bossenno appartient donc sans conteste à ce premier sous-groupe.

On peut comparer la pièce triangulaire de Carnac, pourvue d'un axe de symétrie, à une plaque articulée, appartenant peut-être au sous-groupe b, découverte en surface dans le fort du Litus Saxonicum de Rutupiae à Richborough (Kent, Grande-Bretagne) [Cunliffe, 1968,

6. Mais Suzuki considère, à tort, que ces deux pièces relèvent du Quoit Brooch Style, du début de l'époque anglo-saxonne, bien que, dans une note publiée en 1996 (Ager, 1996), nous ayons clairement montré qu'elles appartenaient à un style et une époque différents.

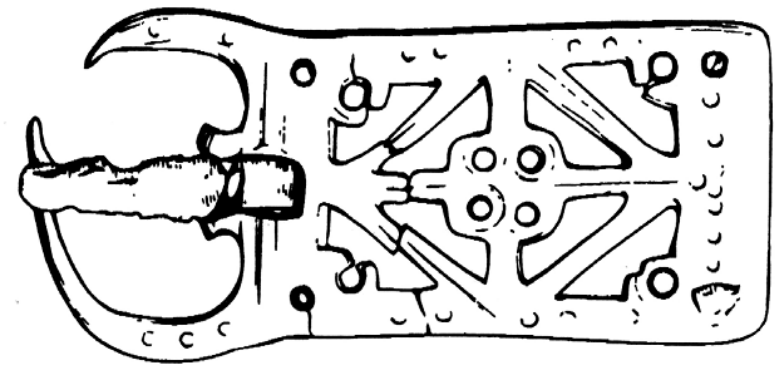

Figure 4 : Plaque-boucle de Portway en Andover (Hampshire, G.-B.) (L. = $6,5 \mathrm{~cm}$ ) (d'après Cook, Dacre, 1985).

Figure 4: Belt buckle from Portway in Andover (Hampshire, G.-B.) $($ L. $=6,5 \mathrm{~cm}$ ) (after Cook, Dacre, 1985).

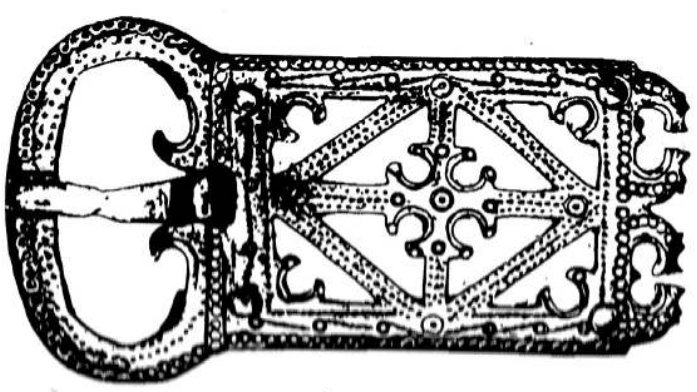

Figure 5 : Plaque-boucle d'Amiens (Somme) (L. = 6,6 cm) (d'après V. Evison, 1965).

Figure 5: Belt buckle from Amiens (Somme) (L. =6,6 cm) (after V. Evison, 1965).

p. 94, pl. 35, 104; fig. 6]. Selon Alison Cook, l'objet original fut probablement brisé en deux au cours de l'Antiquité et réutilisé. Deux triangles ajourés devaient y être placés base contre base, dessinant un losange à l'intérieur d'un cadre rectangulaire, comme dans les autres pièces du groupe Amiens-Andover où ce losange est cependant parfois dépourvu de cet encadrement.

Le thème de l'anille cruciforme que l'on voit sur la boucle d'Amiens apparait aussi sur une plaque-boucle articulée, découverte dans le cimetière anglo-saxon d'Alfriston (East Sussex, Grande-Bretagne), où, dans la tombe n ${ }^{\circ} 103$, probablement celle d'une femme, elle était portée à la taille et associée aux éléments d'une châtelaine, ainsi que sur une pièce, sans provenance connue, mais vraisemblablement mise au jour en Angleterre et conservée dans la collection de M. Stuart Laycock (Evison, 1965, fig. 23g; Suzuki, 2000, p. 127-128, no 4, pl. 4, fig. 4, que l'auteur, une fois encore, attribue au Quoit Brooch Style; Welch, 1983, p. 91, fig. 40a; communication personnelle de S. Laycock, du 20/03/2019). Bien que la théorie d'une invasion du sud de l'Angleterre par les Francs au cours du ve s., proposée par Vera Evison, ne soit plus acceptée aujourd'hui, il n'en reste pas moins probable qu'un petit nombre de Francs 
s'installa dans la région pendant l'époque post-romaine (Chadwick Hawkes et Dunning, 1961, 40).

Les paires de têtes animales affrontées, comme celles apparaissant sur l'objet des Bossenno, sont caractéristiques des deux sous-groupes où elles sont d'ordinaire placées aux extrémités de la plaque, comme on le remarque, par exemple, sur les plaques-boucles d'Alfriston et d'Amiens (voir supra), et sur une boucle - ou plaque-boucle - du sous-groupe b, réputée provenir du nord de la France, sans autre précision (Ager, 1996, fig. 1) [fig. 7], ainsi que sur une plaque-boucle articulée à décor complexe, appartenant au même sous-groupe, mise au jour dans les niveaux de destruction (" état 2 ») des thermes de Tours. Sur cette plaque-boucle, le cou des animaux se prolonge en motifs delphiniformes et la partie centrale est ornée de quatre panneaux ajourés de forme carrée, contenant des croix de Saint-André avec quadrilobes centraux (Galinié, 1999, fig. 11; Motteau, 2007, fig. 28a). Une boucle fragmentaire, appartenant peut-être au sousgroupe b, découverte à Frilford (Oxfordshire, GrandeBretagne), porte également ce qui semble être des têtes animales (Evison, 1968, fig. 3d). Des têtes semblables se voient aussi sur les côtés de la boucle et de la plaque d'une plaque-boucle à double ardillon, mais d'un modèle différent, provenant d'Arras (Evison, 1968, p. 237, fig. 4h). Comme le souligne avec raison Vera Evison, ce thème des têtes animales affrontées est d'origine continentale, comme le sont d'ailleurs les doubles ardillons sur des boucles de la fin du Iv s. (Böhme, 1974, p. 67; Böhme, 1986, Abb. 11-12), éléments qui apparaissent également dans la variante Evison b des séries de boucles aux dauphins affrontés, communes dans les provinces occiden-
Figure 6 : Fragment de plaque-boucle de Richborough (Kent, G.B.) (L. conservée $=2 \mathrm{~cm}$ ) (d'après $B$. Cunliffe, 1968).

Figure 6: Belt buckle fragment from Richborough (Kent, G.-B.) (preserved L $=2 \mathrm{~cm}$ ) (after B. Cunliffe, 1968). tales à l'époque romaine (Evison, 1981, p. 129-130). Les boucles d'époque romaine présentant des ardillons volutés, comme celle d'Arras, sont un type pérenne, apparaissant en Grande-Bretagne et sur le continent entre la fin du i ${ }^{\text {er }}$ s. apr. ou le début du siècle suivant et le $\mathrm{IV}^{\mathrm{e}}$ s. Elles se rencontrent ainsi en Cumbria, en Espagne, à Krummensee, Eutin, à la Saalburg et autres sites du limes allemand, et, plus loin encore, dans les Balkans, en Chersonèse et au Moyen-Orient (Evison, 1968, fig. 3a; base en ligne du Portable Antiquities Scheme sur le site finds.org.uk, ID, référence LANCUM-123688; Pinar Gil 2017, p. 130, n 7 , fig. 22 en haut à droite et 44A; Oldenstein 1976, p. 51 et sq., p. 57, Taf. 74 et 76.1011; Sommer 1984 , Taf. $13.1-4,14.3$ et $5,15.3$ et 16.2 , carte p. 105; Soupault 2003, pls. 2.4, 11.1 et 3), sans que l'on sache exactement pourquoi Vera Evison considère la boucle d'Arras comme une copie franque du début du $\mathrm{VI}^{\mathrm{e}}$ s. plutôt que comme un objet de l'Antiquité tardive. Il pourrait, il est vrai, s'agir d'un objet transmis d'une génération à l'autre.

Les autres exemples du groupe Amiens-Andover comprennent une boucle sans provenance mais vraisemblablement d'origine anglaise, de la collection Laycock, une autre boucle, également sans provenance, mais ayant pro-

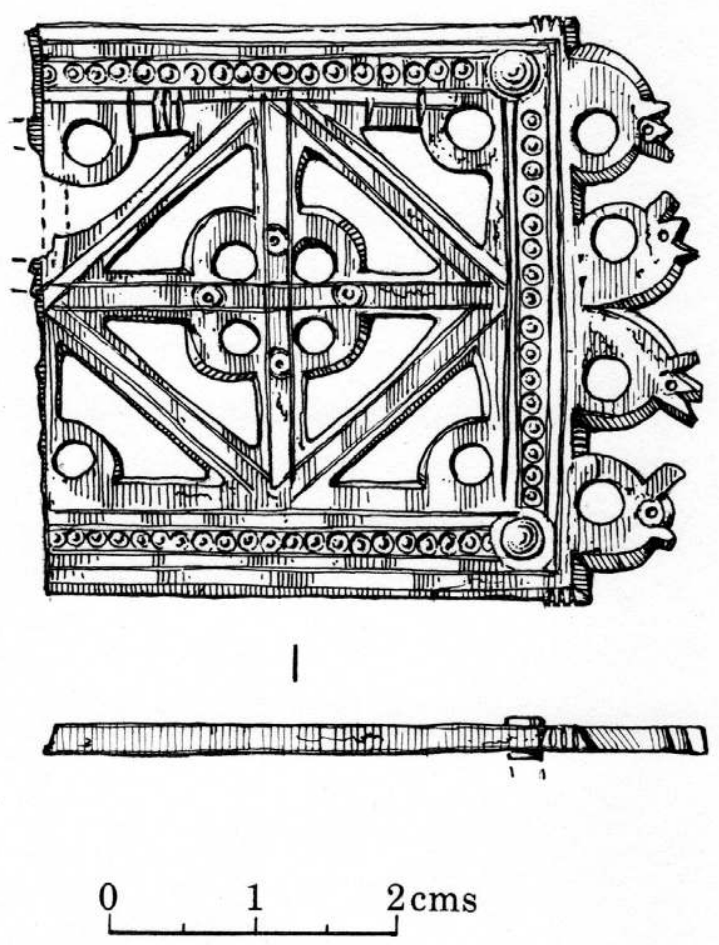

Figure 7 : Fragment de plaque-boucle, probablement du nord de la France (d'après B. Ager, 1996).

Figure 7: Belt buckle fragment probably from the north of France (after $B$. Ager, 1996). 
bablement appartenu autrefois à une collection anglaise qui aurait été dispersée (Laycock, 2009, fig. 4), et un élément ajouré, en forme de losange, relevant nettement du même ensemble typostylistique, découvert fortuitement à Churchills, sur l'île de Wight (Portable Antiquities Scheme, $\mathrm{n}^{\circ}$ IOW-807C07).

Comme le montrent les découvertes de Tours, Richborough et Carnac, ce groupe appartient à l'Antiquité tardive et trouve peut-être son origine dans les boucles et plaques-boucles dont le thème décoratif, en forme d' "Union Jack ", se compose de rayons jaillissant d'un oculus central (Sommer, 1984, Abb. a).

Les plaques-boucles de type provincial d'un seul tenant, comme c'est le cas des exemplaires d'Amiens et d'Andover et des deux plaques-boucles de la collection Laycock, sont plus précisément apparues sur le continent dans la première moitié du ve s. (Evison, 1968, p. 236; Böhme, 1974, p. 71-73, Texttafel B, avec datation légèrement revue dans Böhme, 1987, Abb. 40). Il est probable que l'on continua d'en produire en Bretagne insulaire jusqu'à la séparation de l'Empire romain et des civitates de l'île, vers 410 apr. J.-C., tandis qu'en Gaule, il est possible que l'on ait continué d'en façonner dans les décennies suivantes. Les plaques-boucles articulées appartiennent sans doute globalement à la même période, mais semblent typologiquement antérieures aux précédentes. Les exemplaires mis au jour en contexte funéraire, comme à Andover et Alfriston, correspondent vraisemblablement à des réutilisations plus tardives.

Les deux sous-groupes peuvent-ils avoir été produits dans le(s) même(s) atelier(s) ? Pour Vera Evison, les plaques-boucles présentant des têtes animales en saillie étaient façonnées dans le nord de la Gaule (Evison, 1968, p. 237). Mais les deux sous-groupes se rencontrant tant en Angleterre qu'en France, on peut se demander s'ils ne furent pas produits sur des modèles identiques des deux côtés de la Manche. Il n'existe, pour l'instant, qu'un trop petit nombre de ces pièces pour que ces questions trouvent une réponse immédiate. Il faut aussi noter que, contrairement aux garnitures de ceinturon de l'Antiquité tardive décorées en Kerbschnitt et aux pièces associées, mieux connues des chercheurs, les pièces du groupe Amiens-Andover ne se rencontrent pas, du moins à ce jour, dans la région du limes rhénan.

De façon plus générale, on peut observer une parenté stylistique entre les objets du groupe Amiens-Andover et ceux, plaques-boucles et autres, ornés dans le style nonzoomorphe et géométrique dit Quoit Brooch Style, appartenant aux décennies médianes du ve s. (Evison, 1965, p. 65-6; Evison, 1968) et précédant l'adoption, par les Anglo-Saxons et les peuples du nord de la Gaule, du style I de Salin. On signale d'ailleurs la découverte d'un nombre croissant d'objets décorés dans le Quoit Brooch Style ou dans un style voisin, non seulement dans le sud de l'Angleterre mais aussi dans le nord de la France, de la Bretagne armoricaine au Pas-de-Calais. Outre la trouvaille, déjà ancienne, d'une plaque-boucle répondant à ce style et réutilisée dans une tombe du début du vI à Pontde-Buis (Finistère) [Abgrall, 1911; Galliou, à paraittre], il faut en effet signaler la découverte récente, dans la péninsule armoricaine, à Saint-Marcel (Morbihan), Rennes et La Mézière (Ille-et-Vilaine) de toute une série de pièces ainsi ornées (Ager, 1985, p. 11, 16-17; Ager, 1990, p. 154; Soulat, 2009, p. 62-66: Le Boulanger et al., 2012, en particulier p. 240-2; Labaune-Jean, 2015 : Swift, 2019, fig. 21, 23-24). Il est possible qu'il faille ajouter à cette liste une fibule annulaire et un élément tubulaire que l'on a longtemps pensé provenir de la nécropole de Herpes (Charente), mais dont l'origine, bien que française, reste en fait incertaine (Haith, 1988).

Comme on l'a précédemment noté, il convient de clairement différencier les groupes d'ornements de ceinturon du groupe Amiens-Andover de ceux du Quoit Brooch Style, bien que ces deux ensembles partagent certains traits stylistiques, surtout les paires de têtes animales affrontées, et les ardillons formant courtes volutes des boucles, éléments tous deux empruntés à des modèles de l'Antiquité tardive. Le contexte tardo-romain du groupe Amiens-Andover - en laissant pour l'instant de côté les pièces exhumées de cimetières anglo-saxons -, contrairement au contexte post-romain des trouvailles d'objets du Quoit Brooch Style, tend à montrer que le premier de ces ensembles est le plus ancien et qu'il appartient à l'univers de la production d'ornements métalliques dans les provinces au cours de l'Antiquité tardive (Welch, 1983, p. 91). Toutefois, le fait que quatre éléments du groupe Amiens-Andover aient été mis au jour dans des cimetières anglo-saxons semble révéler une certaine coïncidence chronologique - du moins dans leur période d'utilisation - entre les deux ensembles. On ne peut que souhaiter que de nouvelles trouvailles permettent d'établir plus clairement le lien entre ces deux groupes.

\section{UN FRAGMENT DE FERRET DE CEINTURE? (FIG. 8)}

De la pièce $\mathrm{n}^{\circ} 2$ de la butte $\mathrm{F}$ provient un objet brisé en cuivre allié et dont l'interprétation est, dès lors, rendue délicate (Miln, 1877, p. 188 et F, pl. II, nº 2). La partie conservée se compose d'un corps de forme triangulaire, orné de trois cannelures (deux latérales et une médiane) ayant servi de guide pour tracer, au poinçon, des lignes de points, et de deux ocelles, placés de part et d'autre de la ligne médiane. De la partie sommitale se détachent deux ensembles d'excroissances symétriques, formant 


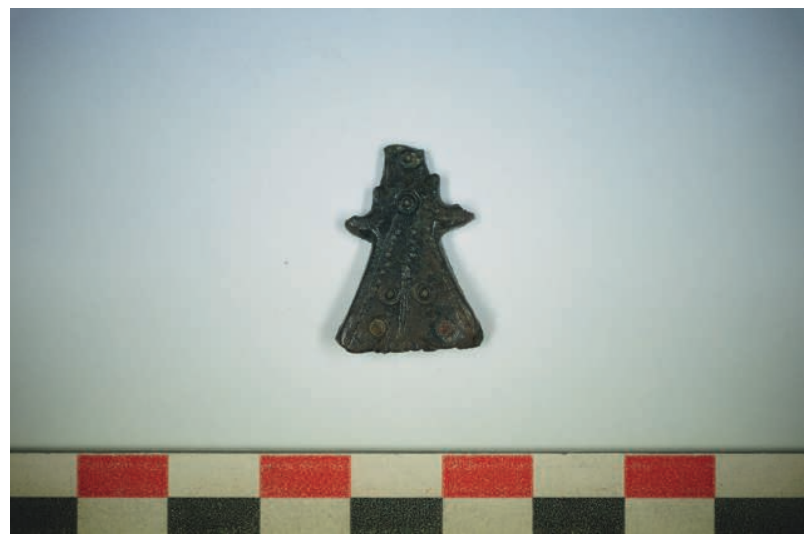

Figure 8: Carnac, Les Bossenno : fragment de ferret de cingulum (?) (cliché : M. Pérez-Bleuzen, Musée de Carnac).

Figure 8: Carnac, Les Bossenno : aglet fragment from a cingulum (?) (photo: M. Pérez-Bleuzen, Musée de Carnac).

volutes ou enserrant peut-être à l'origine un oculus ${ }^{7}$, et doublées d'une ligne semi-circulaire de points, ainsi qu'un axe rectiligne (brisé), orné d'une ligne médiane d'ocelles à double couronne. La partie inférieure - la plus large - de cet objet est creusée d'une profonde gorge latérale qui la subdivise en deux épaisseurs reliées par deux rivets, dont la tête apparaît sur les deux faces. Il pourrait s'agir, sous toutes réserves, d'un ardillon de boucle de cingulum, semblable à celui de boucles de la fin du $\mathrm{IV}^{\mathrm{e}} \mathrm{s}$. de Colchester (Chadwick Hawkes et Dunning, 1961, p. 51, $\mathrm{n}^{\circ}$ 6, fig. 17, $\mathrm{n}^{\circ}$ e) et de Furfooz (Belgique) (Sommer, 1984, Taf. 15, n ${ }^{\circ}$ ), ou, plus probablement d'un ferret en forme d' " amphore » de courroie ou de languette terminale de ceinturon (Sommer, 1984, Taf. 19, $\mathrm{n}^{\text {os }} 5-14$ ). Si l'on admet cette dernière hypothèse, ce type d'objet, assez largement répandu dans le monde romain (Simpson, 1976, p. 198-199, fig. 4; Nuțu, 2011, p. 187-188), est bien daté des années 350-420 apr. J.-C.

\section{LA FIBULE PENANNULAIRE DE TYPE FOWLER C2 (FIG. 9)}

Dans la pièce 8 de la butte $C$ du bâtiment thermal fut mise au jour une fibule penannulaire ${ }^{8}$ en cuivre allié, d'un diamètre d'un peu plus de $30 \mathrm{~mm}$ (Miln, 1877, C, 125 et pl. III, 127, $\mathrm{n}^{\circ} 3$ ). L'arc, plat, aux extrémités repliées sur elles-mêmes en courts enroulements striés, est de section quadrangulaire et orné d'une frise d'esses couchées

7. Le dessin de l'objet donné par James Miln (op. cit., pl. F II, fig. 2) montre que le possible oculus droit était plus complet lors de la découverte.

8. L'appellation « fibule en oméga », en raison de la forme de l' $\Omega$ grec, ne correspond, en fait, qu'à un seul des sous-types des fibules penannulaires (c'est-à-dire des fibules dont l'arc est en forme d'anneau ouvert), soit le type B d'Elizabeth Fowler (2014). On notera d'ailleurs qu'une fibule de ce dernier type a été découverte dans la villa

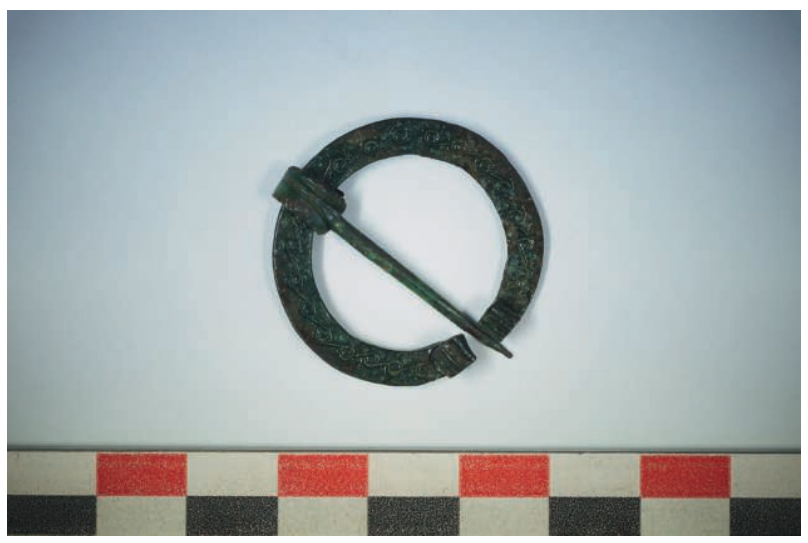

Figure 9 : Carnac, Les Bossenno : fibule penannulaire Fowler C2 (Cliché : M. Pérez-Bleuzen, Musée de Carnac).

Figure 9: Carnac, Les Bossenno : penannular brooch Fowler C2 (photo : M. Pérez-Bleuzen, Musée de Carnac).

et enchaînées, liées par des ocelles. L'ardillon est conservé, en place.

Cet objet relève de la classe C d'Elizabeth Fowler, que celle-ci avait d'abord datée, dans son ensemble, du début de l'époque romaine (Fowler, 1960, p. 165-166). En 1977, étudiant la fibule de la tombe collective de Sablesd'Or-les-Pins en Pléhérel-Fréhel (Côtes-d'Armor), nous avions pu montrer qu'une grande partie des pièces de ce type appartenait en fait à l'Antiquité tardive (Galliou, 1977), ceci étant admis, quelques années plus tard, par Elizabeth Fowler dans son étude des fibules penannulaires mises au jour à Colchester (Fowler, 1983, p. 19; voir aussi Höck, 2013, Form 2a3 et Fundliste 2a, p. 338; Booth, 2014, p. 145-146). L'arc de la plupart de ces fibules n'est pas orné, sinon parfois d'un décor de chevrons pointés assez sommaire, comme à Colchester (Fowler, 1983, p. 18 et fig. 16, nº 102), Trêves (Cüppers, 1984, p. 302 et fig. 156, 1), Lavoye (Meuse) [Chenet, 1926, p. 237-238, fig. 1. E], Le Hérapel en Cocheren (Moselle) [Bergthol, 1929, p. 107, L] ou Brébant (Marne) [Le Clert, 1898, p. $62, n^{\circ} 165$ et pl. XVIII, no 165$]^{9}$ (fig. 10). Le décor de la pièce de Carnac, quant à lui, se voit aussi sur des plaques-boucles de Upper Upham, Aldbourne (Wiltshire, Grande-Bretagne), Cirencester (Gloucestershire, GrandeBretagne) et Duston (Northamptonshire, GrandeBretagne) [Chadwick Hawkes et Dunning, 1961, fig. 13, g; fig. 15, n-o], mais aussi sur la frise entourant la panse de l'un des pichets en poterie, sans doute tardifs, mis au jour dans la villa des Bossenno (Miln, 1877, B pl. XIII, $\mathrm{n}^{\circ} 1$ ). Notons enfin que le tableau publié par Anton Höck (Höck, 2013, tabl. 1) des trouvailles de fibules de ce type dans les sépultures montre qu'elles étaient très majoritaire-

9. Notons que la fibule penannulaire du cimetière de Merteville (Loizel et Coquelle, 1977, p. 183, n 71/18/06 et fig. 96 [tombe 71/18]) était ornée à la feuille d’or. 


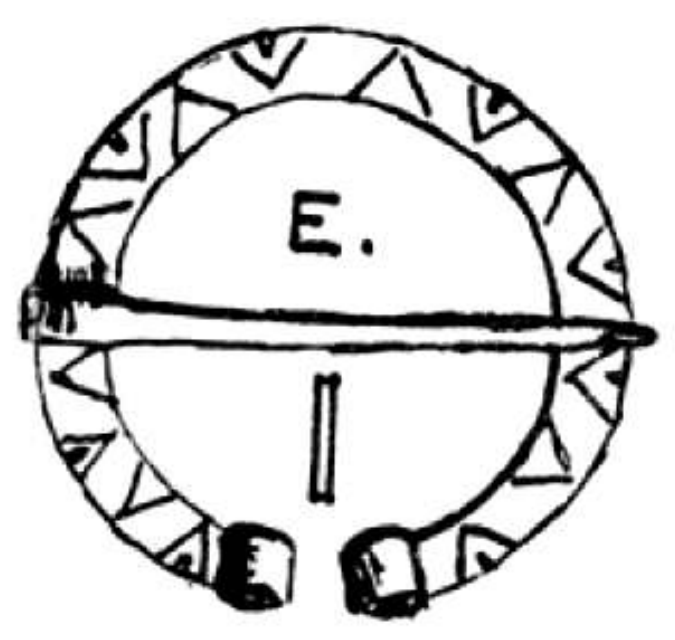

Figure 10 : Fibule penannulaire à décor de chevrons (Lavoye, Meuse) (diam. $=3,2 \mathrm{~cm}$ ) (d'après G. Chenet, 1926).

Figure 10: Penannular brooch with chevron motif (Lavoye, Meuse) (diam. = 3,2 cm) (after G. Chenet, 1926).

ment portées, plutôt par des hommes que par des femmes (Höck, 2013, p. 351, tabl. 2-3), sur l'épaule droite, très probablement pour fixer le pli d'un vêtement, manteau ou autre. Comme les fibules cruciformes, elles paraissent être un insigne de fonction ou de rang, et, selon Erwin Keller (1971, p. 55-56), elles appartiendraient à l'uniforme des militaires de l'Antiquité tardive.

Ce type d'agrafe est assez largement répandu, des îles Britanniques à la Pannonie (Höck, 2013, Abb. 3, qui prend en compte tous les sous-types; Fundliste 2a) et, en Gaule, se rencontre essentiellement dans le Bassin parisien, en Gaule Belgique et dans la péninsule armoricaine. Cinq ou six sites de la péninsule, Alet en SaintMalo (Galliou, 1975, p. 81), Carnac, Corseul (Le Cloirec, 2001, p. 73-74, fig. 20, no 112), La Hérupée en Guer (Petit, 1970, p. 277, sépulture no 2), Sables-d'or-les-Pins en Pléhérel-Fréhel (Galliou, 1977) et peut-être GorréBloué en Plouescat (Finistère) [Abgrall, 1919, p. 46] ${ }^{10}$, ont en effet livré des fibules de ce modèle, bien datées de l'Antiquité tardive lorsque le contexte de leur découverte est connu.

S'il est impossible, en l'absence de toute donnée stratigraphique, de dater précisément l'exemplaire découvert aux Bossenno, il apparaît nettement, néanmoins, que les fibules du type Höck 2a sont datées, dans l'Est de l'Europe (contextes funéraires), d'une période couvrant la seconde moitié $\mathrm{du} \mathrm{IV}^{\mathrm{e}} \mathrm{s}$. et les premières décennies

10. L’abbé Jean-Marie Abgrall y signale la découverte d'une « boucle ronde de forme circulaire, avec son ardillon, tout son pourtour gravé de guillochures ». La fibule de type C, découverte à Mané-Véchen, en Plouhinec (Morbihan) [information A. Provost] est d'un modèle à arc de section circulaire, sans doute plus ancien. du siècle suivant (Höck, 2013, tabl. 1). C’est la même chronologie que l'on observe en Gaule, comme à Lavoye (Meuse) - avec monnaies dont les plus récentes sont d'Arcadius (395-408 apr. J.-C.) [Chenet, 1926, p. 237-238, fig. 1, E], Merteville (Aisne) [seconde moitié du $\mathrm{IV}^{\mathrm{e}} \mathrm{s}$.] (Loizel et Coquelle, 1977, nº 71/18/06 et fig. 96), Liévin (Pas-de-Calais) [fin du Iv s. soger, 1967, p. 751 et fig. 8), Matagne-la-Grande-Doische (Belgique, province de Namur) [330-402 apr. J.-C.] (Rober, 1993, p. 29, fig. 53, n ${ }^{\circ} 15$ ), Pont-sur-Yonne (Yonne) [fin du IV ${ }^{e}$ s. ou début du siècle suivant] (renseignement : M. Feugère). À La Hérupée en Guer (Morbihan), la sépulture n 2, qui a livré une fibule de ce modèle, contenait aussi un gobelet conique en verre de type Isings 106d (Isings, $1957=$ Rütti, 1990, type AR 69) intact de la seconde moitié du $\mathrm{IV}^{\mathrm{e}} \mathrm{s}$. ou du début du siècle suivant, tandis que, dans la tombe voisine $\left(\mathrm{n}^{\circ} 1\right)$, se trouvait une monnaie de Valens (367-375 apr. J.-C.).

\section{SYNTHÈSE}

Le premier enseignement que l'on peut tirer de la présence de ces objets dans la villa des Bossenno est que cet établissement rural fut occupé jusqu'aux premières décennies $\mathrm{du} \mathrm{v}^{\mathrm{e}} \mathrm{s}$. au moins, soit plus tardivement qu'on le pensait généralement en se fondant sur les monnaies les plus récentes (Constance II [337-361] et Magnence [350353]) qui y furent découvertes par Miln (1877, p. 129, 152-153). Il convient, en fait, de revenir sur l'hypothèse émise voici une quarantaine d'années par l'un d'entre nous d'un abandon quasi général des villae de la péninsule armoricaine avant la fin du Iv es. (Galliou, 1983, p. 277). Bien que les monnaies postérieures à 360 , et en particulier celles de la dynastie théodosienne (392-455 apr.) soient particulièrement rares dans la région, où, dans les campagnes, on ne les rencontre que dans la villa du ClosLory en Plouasne (Côtes-d'Armor) [Levitre, 1969, n. p. : monnaie de Théodose frappée en 395] et dans celle de la pointe de la Garenne au Hézo (Morbihan) [Daré, 2014a, p. 156-158 : sept solidi, de Valentinien à Honorius], la présence, dans certains de ces établissements (Kerran en Arradon [Morbihan] : Daré, 2014b, p. 65; Le Pérennou en Plomelin [Finistère] : Arramond, 2009, p. 54-55), de quelques fragments de céramiques à décor estampé, ("Dérivées des sigillées paléochrétiennes du groupe atlantique » = DSP), produites en Aquitaine au $\mathrm{v}^{\mathrm{e}}$ s., sinon plus tard (Rigoir et al., 1973), d'un peigne en os tardif (ManéBourgerel en Arradon [Morbihan] : Daré, 2014c, p. 69 et fig. 30) ainsi que quelques datations ${ }^{14} \mathrm{C}$ y traduisent une occupation de ces lieux au $\mathrm{v}^{\mathrm{e}}$ s., dont la nature et l'amplitude nous échappent néanmoins dans une large mesure. 
Il convient par ailleurs, dans une très large mesure, de dissocier pièces de parure métalliques de l'Antiquité tardive (boucles et plaques-boucles, fibules, etc.) et identité ethnique ${ }^{11}$. Bien que les objets du groupe «AmiensAndover " paraissent surtout se rencontrer dans le Sud-Est de l'actuelle Angleterre, leur présence en Gaule n'a très vraisemblablement aucun rapport avec ce que l'on a coutume d'appeler les " migrations bretonnes", pas plus qu'on ne saurait voir, dans le mobilier des sépultures du petit cimetière de l'Antiquité tardive de Ty-Korn en Gouesnach (Finistère), la preuve de l'implantation, en ce lieu, d'un contingent germanique ou germanisé (Colleter et al., 2012). Notons d'ailleurs, à propos de la pièce de Carnac, que la zone de répartition des objets du groupe "Amiens-Andover " n'est aucunement celle d'où sont censés venir les Bretons qui s'installèrent en Armorique.

On peut en fait penser que la présence de ces boucles et plaques-boucles en Gaule, du moins si l'on admet qu'une partie d'entre elles fut « importée » de Bretagne insulaire, s'explique par la continuité des échanges maritimes dans la Manche et l'Atlantique, dont témoigne, par exemple, la diffusion des DSP en Armorique, où elles se rencontrent aussi à Vannes (église Saint-Patern, rue Sainte-Catherine : Daré, 2014d, p. 367, 373) et à Locmariaquer (Morbihan) [Brunie, 2012, 523], et, plus au nord, en Normandie (Cherbourg, Bayeux), dans le Bassin parisien (Chaidron, 2008) et en Bretagne insulaire (Thomas, 1981; Campbell, 2007). Il est d'ailleurs probable que ces céramiques ne formaient qu'une partie de cargaisons composées, pour l'essentiel, de vins du Sud-Ouest. À ces trafics s'ajoutèrent, à une date plus tardive, ceux reliant la Méditerranée orientale et les îles Britanniques (Fulford, 1989) et qui affectèrent, du moins épisodiquement, certains sites des Côtes-d'Armor comme l'île Lavret en Bréhat (Giot et Querré, 1985) et le Yaudet en Ploulec'h (Cunliffe et Galliou, 2007, p. 38). C’est dans ce contexte général qu'il faut sans doute placer les objets de Carnac.

On ne peut enfin manquer de s'interroger sur la signification de la présence, dans un établissement civil à vocation sans doute essentiellement agricole, d'éléments métalliques que l'on place d'ordinaire au rang des militaria. Notons tout d'abord que, dans l'ouest de la France, bien que peu nombreux, d'autres sites du même type ont eux aussi livré des objets de cette catégorie. C'est ainsi le cas de la villa du Grand-Teil à Avoise (Sarthe) [plaqueboucle et ferret de ceinture " en amphore »] (Lambert, Rioufreyt, 1976, pl. 19, no 2 ), et de celles de Villeneuveen-Drain à Drain (Maine-et-Loire) [boucle de cingulum] (Pape, 1960, p. 74-75; Galliou, 1979-1980) et de

11. Voir, en particulier, pour les questions d'ethnicité : HALSALL G., Barbarian Migrations and the Roman West, 376-568, Cambridge, Cambridge University Press, 2007, p. 35-62.
Kéradennec en Saint-Frégant (Finistère) (éléments de cingulum) [Sanquer et Galliou, 1972, fig. 14, $\mathrm{n}^{\circ} 701$ b1-5], des "boucles de ceinturon ", non décrites et dont on ne sait donc si elles appartiennent bien à l'Antiquité tardive, ayant été découvertes sur d'autres sites ruraux d'époque romaine, mais de nature indéterminée, comme Bodivo en Saint-Barthélemy (Morbihan) [Galliou et al., 2014, p. 298], Saint-Michel en Carnac (Miln, 1877, p. 218219) et Castel Kerandroat en Plésidy (Côtes-d'Armor) ([Bizien-Jaglin et al., 2002, p. 228]. D'autres boucles, enfin, proviennent d'une tombe isolée (dunes d'Étel [Morbihan] : Jacq, 1942) et d'un cimetière de l'Antiquité tardive (Ty-Korn en Gouesnac'h : Colleter et al., 2012, fig. 16, 2.1 et p. 325-326).

Contrairement à ce que l'un d'entre nous avait supposé voici bon nombre d'années (Galliou, 1983, p. 268), il paraît aujourd'hui hautement improbable que ces boucles, plaques-boucles et fibules tardives mises au jour sur des sites ruraux de l'ouest de la Gaule aient fait partie de l'équipement de militaires postés dans les campagnes et relevant d'un réseau de défense qui serait venu compléter les forts et places fortifiées établis sur les côtes. Un tel réseau secondaire, jouant le rôle de filet de rattrapage pour capturer ou tuer les "pirates " qui auraient échappé aux troupes stationnées dans les places-fortes n'a tout bonnement jamais existé (Galliou et Simon, 2015, p. 152-161). Le même phénomène s'observe d'ailleurs en Bretagne insulaire, comme l'a bien montré Douglas Carr : les trouvailles de plaques-boucles tardives y sont plus nombreuses sur les sites ruraux que sur les sites militaires (Carr, 2019, fig. 2), et, dans le sous-ensemble rural, plus fréquentes dans les villae que dans les habitats groupés (ID., ibid., fig. 3). Or, il est essentiel de souligner que, dans l'Antiquité tardive, le cingulum, tout comme les fibules cruciformes (Van Thienen, 2016) et peut-être aussi les fibules Fowler C2, est un signe extérieur de rang et d'autorité (Perez Rodriguez-Aragon, 1992; Feugère, 1996, p. 275-278 ; Schopphoff, 2009). Ainsi, les expressions latines cingulum ponere et cingulum deponere en vinrent-elles à signifier " entrer dans le service civil » et " quitter " celui-ci (Jones, 1964, p. 566) et on rappellera aussi qu'en 298, le futur saint Marcellus de Tanger, centurion de la legio VII Gemina Pia Felix, affichant son christianisme en refusant de participer à une parade organisée pour l'empereur Maximien, aurait jeté à terre ses armes et son cingulum (de Gaiffier, 1943). Notons enfin que les fresques d'une tombe monumentale du début $\mathrm{du} \mathrm{IV}^{\mathrm{e}}$ s. découverte à Silistra (Durostorum), en Bulgarie, montrent deux domestiques apportant cérémonieusement à leur maître, manifestement un homme riche et puissant, les insignes de son rang, l'un tenant un cingulum décoré d'éléments métalliques dorés, l'autre une chlamyde ornée 
d'une fibule cruciforme en or (Atanasov, 2009, p. 449450, fig. 9-10).

La présence, dans l'Ouest comme dans d'autres régions de Gaule, d'objets de ce type sur des sites ruraux et hors de tout contexte militaire est en fait directement liée à l'évolution de la société du temps. La fin de l'époque romaine est en effet marquée, en Gaule et ailleurs, par un accroissement de violence, tant externe (pression exercée par les "barbares " sur les frontières de l'Empire) qu'interne (guerres fratricides entre les prétendants à la pourpre, révoltes sociales, etc.), se doublant d'un lent retrait de l'intervention des autorités impériales et poliades. Dans cet environnement en plein délitement politique et social, les domaines agricoles, fournissant aux populations du voisinage le travail et la nourriture qui leur permettaient de vivre, constituaient nécessairement des îlots de relative stabilité, à condition bien sûr qu'ils soient défendus contre les pillards et les ennemis de tout ordre. En l'absence d'unités officielles de protection, comme celles que les autorités de la civitas ou de la province pouvaient mobiliser lors des siècles précédents, la sécurité des personnes et des biens, du moins sur le domaine et ses environs immédiats, devait être assurée par le personnel, les proches et les clientes et esclaves du propriétaire, formant une petite armée privée - ce que Keith Branigan qualifie de "home guard» (1977, p. 105; voir aussi Van Ossel, 1995) -, dirigée par le domanier, ce dernier pouvant également se voir aussi confier, par ce qui subsistait des autorités locales, la police des campagnes et des voies de communication, menacées par un brigandage endémique, comme nous l'apprennent plusieurs inscriptions du Haut-Empire (CIL XIII, 289, 2282, 2667, 3689) et le soulignent Peter Brown (1992, p. 126) et Laurent Lamoine (2009, p. 289-371), cet état de fait étant d'ailleurs confirmé, pour l'Antiquité tardive, par Ammien Marcellin (Res gestae XXVIII, 2, 10) et Ausone (Epistolae, IV). L'existence d' "armées " privées est bien attestée, au $v^{e}$ s., par diverses sources, comme par exemple la Vie de Daniel le Stylite ( $\$ 60$ : « le saint empereur Léon entendit parler par beaucoup de ses proches d'un dénommé Titus, homme puissant qui habitait la Gaule et avait à son service nombre d'hommes bien entrainés pour la bataille ") et par Sidoine Apollinaire (Epistolae, III, 1), qui relate comment le sénateur arverne Ecdicius défit les Wisigoths d'Euric devant Clermont-Ferrand à la tête de ses dix-huit cavaliers. En 468, les empereurs d'Orient Léon I ${ }^{\text {er }}$ (457474) et d'Occident Anthémius (467-472) interdisaient aux particuliers d'avoir à leur service des bucellarii ou des esclaves armés, et les propriétaires ne devaient pas entretenir sur leur domaine ou près d'eux des dépendants en armes (armata mancipia seu bucellarios) dont ils pourraient se servir pour causer des troubles ou attaquer leurs voisins (Codex Justinianus, IX, 12, 10), la loi essayant très certainement de corriger un comportement très répandu à cette époque. Qu'ils se soient vus confier ce droit ou qu'ils se le soient arrogé, ces mêmes domaniers pouvaient aussi rassembler, armer et mener au combat des milices paysannes, ainsi que le montrent, pour une autre région, les lettres de Synésios de Cyrène (v. 370-v. 414), évêque de Ptolémaïs, rapportant comment les autochtones, lassés de l'incompétence et de la corruption des troupes régulières $\mathrm{du}$ dux Libyarum, s'armèrent pour résister aux raids des nomades ausuriens (Seston, 1980, p. 289).

Il paraît donc assez logique de penser que ces objets de parure, fibules et éléments de cingulum, signes de prestige et de pouvoir ${ }^{12}$, aient été portés par des propriétaires terriens, petits "seigneurs " exerçant leur pouvoir localement, ce phénomène n'étant d'ailleurs pas particulier à la Gaule de l'Ouest si l'on en croit la découverte de boucles de cingulum dans des villae du Centre-Est de la Gaule (Dulphey en Mancey [Saône-et-Loire], La LavièreMonsieur en Joux-la-Ville [Yonne] : Kasprzyk, 2005, p. 308, 392-393) et de l'Hérault (La Vérune en Néffiès : Feugère, 2002, p. 83-84). Une partie de ces objets devait être " importée ", d'autres étant vraisemblablement des productions locales, peut-être façonnées à la demande, les pièces de Néffiès et de Joux-la-Ville étant inachevées, tandis qu'à Ickham, dans le Kent, un établissement rural produisait, entre autres, des pièces de cingulum (Ager, 2010). Notons enfin que, contrairement à ce qu'a observé Stuart Laycock pour la Bretagne insulaire, où les différences de style entre les boucles de cingulum semblent correspondre à des productions à l'échelle de la civitas (Laycock, 2008, p. 154), révélant l'origine géopolitique du porteur, rien de tel n'apparaît pour l'instant dans la région ici étudiée.

\section{Remerciements}

Nous remercions très vivement $\mathrm{C}$. Cornet et $\mathrm{M}$. PérezBleuzen (Musée de Carnac) des photographies des objets étudiés dans ce qui suit.

\section{Bibliographie}

Abgrall J.-M., 1911, "Sépulture romaine découverte à Pontde-Buis ", Bulletin de la Société archéologique du Finistère, 38, p. 188-193.

12. AmORY P., 1997, People and Identity in Ostrogothic Italy, Cambridge, Cambridge University Press, p. 341, rappelle les lois tardives interdisant aux civils de porter l'« uniforme» militaire - les armes, mais aussi le cingulum et le manteau - à Rome, de telles lois ne pouvant viser que des faits existants et donc des phénomènes de revendication ou de saisie de pouvoirs locaux semblables à ceux visibles en Gaule du Nord. 
Abgrall J.-M., 1919, "Établissement gallo-romain de GorréBloué à Plouescat ", Bulletin de la Société archéologique du Finistère, 46, p. 32-48.

Ager B. M., 1985, "The smaller variants of the Anglo-Saxon quoit brooch ", Anglo-Saxon Studies dans Archaeology and History, 4, p. 1-58.

Ager B. M., 1990, "The alternative quoit brooch: an update ", dans Southworth E. (dir.), Anglo-Saxon cemeteries: A reappraisal, Liverpool, National Museums and Galleries on Merseyside, p. 153-161.

Ager B. M., 1996, "A late Roman buckle- or belt-plate in the British Museum, said to be from northern France ", Medieval Archaeology, 40, p. 206-211.

Ager B. M., 2010, "Typology, dating and producing "military" belt-fittings ", dans Bennett P., Riddler I., SpareyGreen C. et al., The Roman Watermills and Settlement at Ickham, Kent, Canterbury, Canterbury Archaeological Trust, p. 152-165.

Arramond J.-C., 2009, Plomelin, Finistère 29. Les thermes du Pérennou. Rapport de fouille archéologique programmée annuelle, 19 mai-13 juin 2008, Léojac, Auteur.

Atanasov G., 2009, "Late antique tomb in Durostorum-Silistra and its master", Revista Pontica, 40, p. 447-468.

Bergthol E., 1929, "Fibules gallo-romaine du Mont-Hérapel (Moselle) ", Revue des musées, nº 22, p. 106-108.

Bizien-Jaglin C., Galliou P., Kerébel H., 2003, Carte archéologique de la Gaule: Les Côtes-d'Armor, Paris, Académie des Inscriptions et Belles-Lettres, 331 p.

Вӧнме H. W., 1974, Germanische Grabfunde des 4. bis 5. Jahrhunderts zwischen unterer Elbe und Loire, Münich, Münchner Beiträge zur Vor-und Frühgeschichte, 384 p.

Böнme H. W., 1986, « Das Ende der Römerherrschaft in Britannien und die angelsächsische Besiedlung Englands im 5. Jahrhundert ", Jahrbuch des Römisch-Germanischen Zentralmuseums Mainz, 33, p. 469-574.

Böнme H. W., 1987, "Gallien in der Spätantike ", Jahrbuch des Römisch-Germanischen Zentralmuseums zu Mainz, 34, p. 770-773.

Воотн A. L., 2014, Reassessing the long chronology of the penannular brooch in Britain: exploring changing styles, use and meaning across a millennium, thèse de doctorat inédite, université de Leicester, 385 p.

Branigan K., 1977, The Roman Villa in South-West England, Bradford-on-Avon, Moonraker Press, 128 p.

Brown P., 1992, Pouvoir et persuasion dans l'Antiquité tardive, Paris, Éditions du Seuil, 253 p.

Brunie I., 2012, "Découverte maritime à Locmariaquer (Morbihan) : nouvelles données sur les échanges en Atlantique pendant l'Antiquité ", Bulletin de la Société française d'étude de la céramique antique en Gaule. Actes du congrès de Poitiers (17-20 mai 2012), p. 521-534.

Campbell E., 2007, Continental and Mediterranean Imports to
Atlantic Britain and Ireland, AD 400-800, York, Council for British Archaeology, 163 p.

CARr D., 2019, "Roman warlords and the early medieval world ", en ligne (consulté le 12 mars 2020).

Chadwick Hawkes S. C. et Dunning G. C., 1961, "Soldiers and settlers in Britain, fourth to fifth century, with a catalogue of animal-ornamented buckles and related belt-fittings ", Medieval Archaeology, 5, p. 1-70.

Chaidron C., 2008, "Note sur la découverte de sigillées paléochrétiennes dans le nord de la France ", Revue archéologique de Picardie, 3-4, p. 247-251.

Chenet G., 1926, "Agrafe circulaire ou fibule en oméga ", Revue des musées et des collections archéologiques, 7, p. 236240.

Colleter R., Labaune-Jean F. et Hinguant S., 2012, " Le cimetière de Ty Korn à Gouesnach (Finistère) ", Gallia, 69/1, p. 309-344.

Cook A. M. et Dacre M. W., 1985, Excavations at Portway, Andover 1973-1975, Oxford, Oxford University Committee for Archaeology, $192 \mathrm{p}$.

Cunliffe B., 1968, Fifth Report on the Excavations of the Roman Fort at Richborough, Kent, Londres, Report of the Research Committee of the Society of Antiquaries, 414 p.

Cunliffe B. et Galliou P., 2007, Les Fouilles du Yaudet en Ploulec'h, Côtes-d'Armor, vol. 3. Le site : Du iv's. apr. J.-C. à aujourd'hui, Oxford, Oxford University Committee for Archaeology, 308 p.

Cüppers H., 1984, Trier, Kaiserresidenez und Bischofssitz, Mayence, von Zabern, 368 p.

Daré S., 2014a, « Le Hézo, La Garenne », dans Galliou P. et al., Carte archéologique de la Gaule : 56 - Le Morbihan, Paris, Académie des Inscriptions et Belles-Lettres, p. 152-158.

Daré S., 2014b, "Arradon, Kerran ", dans Galliou P. et al., Carte archéologique de la Gaule : 56 - Le Morbihan, Paris, Académie des Inscriptions et Belles-Lettres, p. 62-65.

DARÉ S., 2014c, "Arradon, Mané-Bourgerel », dans Galliou P. et al., Carte archéologique de la Gaule : 56 - Le Morbihan, Paris, Académie des Inscriptions et Belles-Lettres, p. 68-70.

Daré S., 2014d, "Vannes ", dans Galliou P. et al., Carte archéologique de la Gaule : 56 - Le Morbihan, Paris, Académie des Inscriptions et Belles-Lettres, p. 338-403.

Evison V. I., 1965, The Fifth-Century Invasions South of the Thames, London, University of London Press, 142 p.

Evison V. I., 1968, "Quoit Brooch Style buckles », Antiquaries Journal, 48, p. 231-246.

Evison V. I., 1981, "Distribution maps and England in the first two phases ", dans Evison V. I. (dir.), Angles, Saxons and Jutes. Essays presented to J.N.L. Myres, Oxford, Clarendon Press, p. 126-167.

Feugère M., 1996, "L'armement du Bas-Empire ", dans Reddé M. (dir.), L'Armée romaine en Gaule, Paris, Éditions Errance, p. 267-278. 
Feugère M., 2002, « Le mobilier militaire romain dans le département de l'Hérault », Gladius, 22, p. 73-126.

Fowler E., 1960, "The origins and development of the penannular brooch in Europe ", Proceedings of the Prehistoric Society, 26, p. 149-177.

Fowler E., 1983, "Penannular Brooches ", dans Crummy N. (dir.), Colchester Archaeological Report 2: The Roman Small Finds from Excavations in Colchester 1971-9, Colchester, Colchester Archaeological Trust Ltd., p. 18-19.

Fowler E., 2014, "The Origin and Development of the Penannular Brooch in Europe ", Proceedings of the Prehistoric Society, 26, p. 149-177.

Fulford M., 1989, "Byzantium and Britain: a Mediterranean perspective on Post-Roman Mediterranean Imports in Western Britain and Ireland ", Medieval Archaeology, 33, p. 1-6.

Gaiffier B. DE, 1943, «S. Marcel de Tanger ou de Léon? Évolution d'une légende ", Analecta Bollandiana, 61, p. 116139.

Galinié H., 1999, "Tours from an archaeological standpoint ", dans Karkov C. E., Wickham-Crowley K. M. et Young B. K. (dir.), Spaces of the Living and the Dead: an archaeological dialogue, American Early Medieval Studies, 3, Oxford, p. 101 et sq.

Galliou P. et al., 2014, Carte archéologique de la Gaule : 56 - Le Morbihan, Paris, Académie des Inscriptions et BellesLettres, $445 \mathrm{p}$.

Galliou P., 1975, "Les objets de parure et de toilette découverts à Alet ", Les Dossiers du Centre régional archéologique d'Alet, 3, p. 77-85.

Galliou P., 1977, « La fibule », dans Bardel J.-P., Galliou P., Giот P.-R. et Рісот S., «Une tombe à sépultures multiples $\mathrm{du}$ Bas-Empire romain découverte aux Sables-d'Or-lesPins en Pléhérel (C.-du-N.) ", Archéologie en Bretagne, 13, p. 29-34.

Galliou P., 1980, "Une boucle de ceinture du Bas-Empire découverte à Drain (Maine-et-Loire) ", Andes, 9, p. 115-118.

Galliou P., 1983, L’Armorique romaine, Brasparts, Les Bibliophiles de Bretagne, $310 \mathrm{p}$.

Galliou P., à paraître, "À propos de la sépulture de l'Antiquité tardive de Goas-an-Eyec à Pont-de-Buis (Finistère) ", Britannia Monastica.

Galliou P. et Simon J.-M., 2015, « Le Castellum de Brest et la défense de la péninsule armoricaine au cours de l'Antiquité tardive ", Rennes, Presses universitaires de Rennes, 220 p.

Giot P.-R. et Querré G., 1985, «Le tesson d'amphore B 2 de l'île Lavret (Bréhat, Côtes-du-Nord) et le problème des importations ", Revue archéologique de l'Ouest, 2, p. 95-100.

Наттн C., 1988, «Un nouveau regard sur le cimetière d'Herpes (Charente) ", Revue archéologique de Picardie, $\mathrm{n}^{\text {os } 3-4, ~ p . ~ 71-~}$ 80 .

Höск A., 2013, "Zu den Ringfibeln mit seitlich aufgerrolten
Enden ", dans Grabherr G. et al. (dir.), Fibeln und Bestandteile der Bekleidung als Mittel zur Rekonstruktion von interregionalem Austausch und zur Abgrenzung von Gruppen vom Ausgreifen Roms während des 1. Punischen Krieges bis zum Ende des Weströmischen Reiches, Innsbrück, Innsbrück University Press, p. 334-399.

Isings C., 1957, Roman Glass from Dated Finds, Groningue, J. B. Wolters, $185 \mathrm{p}$.

JACQ M., 1946, "Une sépulture gallo-romaine », Bulletin de la Société polymathique du Morbihan, p. 9, (procès-verbaux).

Jones A. H. M., 1964, The Later Roman Empire (284-602): A Social, Economic and Administrative Survey, Oxford, Oxford University Press, $522 \mathrm{p}$.

Kasprzy M., 2005, Les Cités des Éduens et de Chalon pendant l'Antiquité tardive, thèse de doctorat inédite, Dijon, 400 p. Keller E., 1971, Die spätrömische Grabfunde in Südbayern, Münich, Beck, 270 p.

Labaune-Jean F., 2015, "Deux fibules du type Quoit Brooch Style datant du v ${ }^{\mathrm{e}}$ s. en Ille-et-Vilaine (Bretagne) ", Cahiers LandArc, 9, p. 1-8.

LAmbert C. et Rioufreyt J., 1976, « La villa gallo-romaine du Grand-Teil à Avoise (72) ", Province du Maine, 78, p. 338370.

Lamoine L., 2009, Le Pouvoir local en Gaule romaine, Clermont-Ferrand, Presses de l'université de ClermontFerrand, 468 p.

LaYcock S., 2008, Britannia, the Failed State. Tribal Conflicts and the End of Roman Britain, Stroud, The History Press, $160 \mathrm{p}$.

LaYcock S., 2009, "Quoit Brooch Style », Treasure Hunting, p. 44 et sq.

Le Boulanger F., Simon L., Jean S., Paitier H., Ager B., Besombes P.-A., Blanchet S., Le Cloirec G. et NaAs P., 2012, " De la ferme antique à la nécropole de l'Antiquité tardive (milieu du $\mathrm{II}^{\mathrm{e}}$ s.-fin du v ${ }^{\mathrm{e}}$ s. apr. J.-C.). Étude archéologique du site de Saint-Marcel "le Bourg"(Morbihan) ", Gallia, 69/1, p. 167-307.

Le Clert L., 1898, Musée de Troyes. Bronzes, catalogue descriptif et raisonné, Troyes, Musée de Troyes, 274 p.

Le Cloirec G., 2001, Les Bronzes antiques de Corseul (Côtesd'Armor), Montagnac, Librairie archéologique, 178 p.

Levitre J., 1969, Site gallo-romain du Clos-Lory, 22-Plouasne. Campagne 1969, rapport de fouille, inédit, non paginé.

Loizel M. et Coquelle J., 1977, « Le cimetière gallo-romain du Bas-Empire de Marteville (02) ", Revue archéologique de Picardie, 4, p. 151-203.

Luco J.-F., 1881, "Sépultures circulaires explorées par James Miln ", Bulletin de la Société polymathique du Morbihan, p. $55-70$.

Luco J.-F., 1883, "Quelques explorations archéologiques de M. Miln ", Bulletin de la Société polymathique du Morbihan, p. $20-35$. 
MacGregor A., 1997, A Summary Catalogue of the Continental Archaeological Collections (Roman Iron Age, Migration Period, Early Medieval), British Archaeological Reports, International Series 674, Oxford, 288 p.

Miln J., 1877, Fouilles faites à Carnac (Morbihan). Les Bosséno et le Mont-Saint-Michel, Paris, Didier, 253 p.

Motteau J., 2007, "Quelques accessoires de vêtement militaire des $\mathrm{IV}^{\mathrm{e}}$ et $\mathrm{V}^{\mathrm{e}}$ s. ", dans Galinié $\mathrm{H}$. (dir.), Tours antique et médiéval: lieux de vie, temps de la ville. 40 ans d'archéologie urbaine, Tours, FERACF, p. 67.

NuȚu G., 2011, "Belt-buckles, strap-ends and appliqués from Halmyris (Moesia Inferior/Scythia) », Novensia, 22, p. 171-199.

Oldenstein J., 1976, «Zur Ausrüstung römischer Auxiliareinheiten: Studien zu Beschlägen und Zieraten der Ausrüstung der römischen Auxiliareinheiten des obergermanisch-raetischen Limesgebietes aus dem zweiten und dritten Jahrhundert n. Chr. ", Bericht der Römisch-Germanischen Kommission, 57, p. 49-284.

PApe L., 1960, «L'établissement gallo-romain de Drain (Maineet-Loire) ", Annales de Bretagne, 67/1, p. 67-76.

Perez Rodriguez-Aragon F., 1992, " Los cingula militiae tardorromanos de la peninsula iberica ", Boletin del Seminario de Estudios de Arte y Arqueologia, 58, p. 239-261.

Petit M., 1970, «Sépultures du Bas-Empire à Guer (Morbihan)", Annales de Bretagne, 77/1, p. 273-278.

Pinar Gil J., 2017, La cronologia dei corredi funerari di epoca visigota in Spagna e Francia meridionale, Rome, BraDypUS, $170 \mathrm{p}$.

Rigoir J., Rigoir Y. et Meffre J.-F., 1973, «Les dérivées des sigillées paléochrétiennes du groupe atlantique ", Gallia, 31, p. 207-263.

Rober A., 1993, Le sanctuaire gallo-romain de Matagne-laGrande, Bruxelles (Archeologia Belgica, 252), 38 p.

Roger F., 1967, "Un cimetière de la fin du Iv ${ }^{\mathrm{e}}$ s. près de la nécropole franque de Liévin ", Revue du Nord, 49, n 195 , p. 741-770.

RüтTi B., 1990, Die römischen Gläser aus Augst und Kaiseraugst, Augst, Amt für Museen und Archäologie des Kantons Basel-Landschaft, $714 \mathrm{p}$.

Sanquer R. et Galliou P., 1972, "Le "château" gallo-romain de Kéradennec en Saint-Frégant (Finistère). Campagnes de fouilles de 1970-1971 ", Annales de Bretagne, 79/1, p. 167-
215.

Scноррноғғ C., 2009, Der Gürtel : Funktion und Symbolik eines Kleidungstück in Antike und Mittelalter, Colognel Vienne, Böhlau Verlag, 324 p.

Seston W., 1980, "Du Comitatus de Dioclétien aux Comitatenses de Constantin ", Scripta varia. Mélanges d'histoire romaine, de droit, d'épigraphie et d'histoire du christianisme, Rome, École française de Rome, p. 284-296.

Simpson C. J., 1976, «Belt-buckles and strap-ends of the Later Roman Empire: A preliminary survey of several new groups ", Britannia, 7, p. 192-223.

Sommer M., 1984, Die Gürtel und Gürtelbeschläge des 4. und 5. Jarhhunderts im römischen Reich, Bonn, Institut für Vorund Frühgeschichte der Rheinischen Friedrich-WilhelmsUniversität, 1665 p.

Soulat J., 2009 (rééd. 2012), Le matériel archéologique de type saxon et anglo-saxon en Gaule mérovingienne, Saint-Germain-en-Laye, Mémoires de l'Association française d'Archéologie mérovingienne, 20, 262 p.

Soupault V., 2003, Les éléments métalliques du costume masculin dans les provinces romaines de la mer Noire: $I I \mathrm{I}^{e}-V^{e} s$. apr. J.-C., Oxford, Archaeopress, 309 p.

Suzuki S., 2000, The Quoit Brooch Style and Anglo-Saxon Settlement, Woodbridge, Boydell Press, 232 p.

Swift E., 2019, "Re-evaluating the Quoit Brooch Style: economic and cultural transformations in the $5^{\text {th }}$ century $\mathrm{AD}$, with an updated catalogue of known Quoit Brooch Style artefacts ", Medieval Archaeology, 63/1, p. 1-55 (avec annexes en ligne).

Thomas C., 1981, A Provisional List of Imported Pottery in PostRoman Western Britain, Redruth, Institute of Cornish Studies, $32 \mathrm{p}$.

VAN Ossel P., 1995, "Insécurité et militarisation en Gaule du Nord au Bas-Empire. L'exemple des campagnes ", Revue du Nord-Archéologie, 77, p. 27-36.

VAn Thienen V., 2016, «A symbol of Late Roman authority revisited: a sociohistorical understanding of the crossbow brooch ", dans Roymans N. et al. (dir.), Social Dynamics in the North West Frontiers of the Late Roman Empire, Amsterdam, Amsterdam University Press, p. 1-29.

Welch M. G., 1983, Early Anglo-Saxon Sussex, Oxford, British Archaeological Reports, British Series 112, 654 p. 
Drei Fundobjekte der römischen Militärausstattung spätantiker Zeit aus der Villa von Bossenno bei Carnac (Morbihan, Bretagne)

Die Untersuchung von drei aus Kupferlegierungen gefertigten Fundobjekten (Elemente eines römischen Militärgürtels, Ringfibel), die während des 19. Jahrhunderts in der Villa von Bossenno bei Carnac (Morbihan, Bretagne) gefunden wurden, hat es gestattet, sie ohne Zweifel in die Spätantike (Ende des 4. Jh. - frühes 5. Jh. n. Chr.) zu datieren. Da derartige Funde üblicherweise als Militaria eingestuft werden, erfordert ihr Auftreten in einer ausschließlich zivilen Siedlung eine genauere Analyse.

Schlagwortindex: Fundobjekte aus Kupferlegierungen, Römische Villenanlage, Spätantike, Militaria.

Tres piezas de ornamento de la Antigüedad tardía de la villa de Bossenno en Carnac (Morbihan)

El estudio de tres objetos metálicos de base cobre (elementos cingulum, fibula anular) descubiertos en el siglo XIX en la villa de Bossenno en Carnac (Morbihan) los sitúa indiscutiblemente en la Antigüedad tardia (finales del siglo IV-principios del siglo V). Se aborda la cuestión del descubrimiento de estos artefactos, generalmente clasificados como militaria, en un asentamiento puramente civil.

Palabras clave: objetos metálicos de base cobre, villa romana, Antigüedad tardía, Militaria. 